\title{
Evolution of the SRGAP2 Gene Is Linked to Intelligence in Mammals
}

\author{
Basant K. Tiwary \\ Centre for Bioinformatics, School of Life Sciences, Pondicherry University, Pondicherry, India
}

\begin{abstract}
What Is It about?
A recent duplication of the gene encoding SLIT-ROBO Rho GTPase-activating protein 2 (SRGAP2) in the primate lineage has been proposed to be associated with the human-specific extraordinary development of intelligence. However, the role of this gene in the manifestation of neural traits related to intelligence in mammals has yet to be established. The evolutionary rate of the SRGAP2 gene is positively correlated with an increase in the number of cortical neurons in mammals, but amino acid replacementsubstitutions are negatively correlated with an increase in the number of neurons. This finding indicates a key role of the SRGAP2 gene in facilitating the evolution of remarkable intelligence in mammals.
\end{abstract}

\section{Key Words}

Brain mass · Neuron number - Encephalization quotient · Purifying selection · Functional constraint

\begin{abstract}
Background/Aims: A recent duplication of the gene encoding SLIT-ROBO Rho GTPase-activating protein 2 (SRGAP2) in the primate lineage has been proposed to be associated with the human-specific extraordinary development of intelligence. There is no report regarding the role of the SRGAP2 gene in the expression of neural traits indicating intelligence in mammals. Methods: A phylogenetic tree of the SRGAP2 gene from 11 mammals was reconstructed using MrBayes. The evolution of neural traits along the branches of the phylogenetic tree was modeled in the BayesTraits, and the $\mathrm{dN} / \mathrm{dS}$ ratio (i.e. the ratio between the number of nonsynonymous substitutions per nonsynonymous site and the number of synonymous substitutions per synonymous site) was estimated using the codon-based maximum likelihood method (CODEML) in PAML (phylogenetic analysis by maximum likelihood). Results: Two neural traits, namely brain mass and the number of cortical neurons, showed statistical dependency on the underlying evolutionary history of the SRGAP2 gene in mammals. A significant positive correlation between the increase in cortical neurons and the rate of nucleotide substitutions in the SRGAP2 gene was observed concomitantly with a significant negative correlation be-
\end{abstract}


tween the increase in cortical neurons and the rate of nonsynonymous substitutions in the gene. The SRGAP2 gene appears to be under intense pressure of purifying selection in all mammalian lineages under stringent functional constraint. Conclusion: This work indicates a key role of the SRGAP2 gene in the rapid expansion of neurons in the brain cortex, thereby facilitating the evolution of remarkable intelligence in mammals.

(C) 2016 The Author(s)

Published by S. Karger AG, Basel

\section{Introduction}

Evolution of intelligence in mammals in general and in primates in particular is one of the greatest puzzles for mankind. There are various attempts to decipher the evolution of intelligence in mammals using phenotypic neural traits such as absolute brain size, encephalization quotient (EQ) and the number of neurons in the cortex [1]. The absolute brain size is the most general property of the brain and measured by brain mass. Intelligence in humans measured in terms of IQ has a low correlation with the head size $(r=0.2)$ [2] and a moderate correlation with intracranial brain volume $(r=0.4)$ [3]. Although a positive correlation is established between the intelligence and overall brain size, intelligence is restricted to some specific regions of the frontal brain [4]. Moreover, there is a strong positive correlation $\left(\mathrm{r}^{2}=\right.$ 0.65 ) between global cognitive ability and the brain size in 24 species of primates [5]. Therefore, these studies clearly indicate that the brain size is a predictive measure of intelligence in mammals. Mammals vary greatly in their body size as well as their brain size. Brain size tends to increase with an increase in body size in mammals due to high metabolic needs, albeit not in equal proportion. Therefore, a better measure of intelligence known as EQ was developed to balance the allometric effects of highly variable body sizes in animals. EQ is a ratio which compares the deviation in brain size of a species in comparison to an expected value based on a standard species [6]. The number of cortical neurons in the brain is calculated using the overall cortical volume and the cell density in the cortex. There is a positive correlation $(\mathrm{r}=0.56)$ between the brain size and the number of cortical neurons in humans [7]. Moreover, this positive correlation is very high $\left(r^{2}=0.97\right)$ between the brain mass and the total number of neurons in 6 species of primates [8]. The number of cortical neurons correlates better with intelligence in mammals than 2 other traits, namely brain size and EQ [1]. EQ seems to be a better predictor of intelligence in comparison to 2 other traits in mammals. Although the average value of EQ is defined as 1 , this value ranges from 0.38 in blue whales to 6.54 in humans [9]. However, the overall brain size was found to be a better predictor of intelligence than EQ and neocortex-based measures in nonhuman primates [5]. All 3 neural traits are found to be approximate predictors of complex cognitive ability in mammals in various studies and were, thus, included in this study for deeper insights into the mechanisms underlying advancement of cognitive ability in mammals.

In a recent study, the SLIT-ROBO Rho GTPase-activating protein 2 gene (SRGAP2) was found to have 2 rounds of human lineage-specific duplications resulting in 4 similar copies of the same gene, namely SRGAP2A-SRGAP2D in humans [10]. These paralogous genes evolved after incomplete duplication transcribed for truncated proteins, which antagonize the function of the parental SRGAP2 protein. This duplication event coincided with the expansion of the neocortex and the emergence of the complex culture and behavior in ancestors of modern humans. It is argued that this duplication of the SRGAP2 gene might have contributed to specific human extraordinary cognitive abilities. It is proven that SRGAP2 has an important role in neuronal migration and differentiation leading to the development of the brain cortex $[11,12]$. Although the duplication event might have triggered a synergistic effect on extraor- 
dinary intelligence in the human brain, the key role of the ancestral SRGAP2 gene in developing cognitive ability in nonhuman primates cannot be ruled out. Thus, a clear understanding of the evolution of this gene in primates with any possible implication on neural traits predicting intelligence will provide a deep insight into the evolutionary mechanism underlying this highly complex trait in humans. Thus, the evolutionary role of SRGAP2 in the progression of intelligence in mammals in terms of 3 neural traits, such as brain size, EQ and number of cortical neurons, can be predicted with the phylogenetic general least-squares (PGLS) framework. This PGLS method has been implemented in the evolution of the ASPM gene, a candidate gene for microcephaly in humans. This gene has a negative correlation with brain size in primates with small body sizes, indicating a vital role for this gene in regulating brain size [13]. This study is the first attempt to explore the relationship between the evolutionary history of the SRGAP2 gene and the evolution of intelligence in mammals using neural traits.

\section{Materials and Methods}

\section{Reconstruction of the Phylogenetic Tree}

Since the primary objective of this study was to decipher the role of the SRGAP2 protein in the development of neural traits as proxies of intelligence, only coding sequences of the $S R G A P 2$ gene were included in this study. The orthologous coding sequences of the SRGAP2 gene from 11 species of mammals were retrieved from Ensembl version 80 (see online suppl. table S1; for all online suppl. material, see www.karger.com/doi/10.1159/000443947). Ensembl has a collection of genomic databases of vertebrates and other eukaryotes with all necessary information required for the evolutionary analysis, such as orthologs, paralogs, gene loss and gene gain. All orthologs of SRGAP2 were subjected to multiple sequence alignment using HyPhy version 2.2 [14]. HyPhy is a popular software for various types of evolutionary analyses, such as codon-based alignment, reconstruction of a phylogenetic tree and testing the molecular clock hypothesis. The best-fitting model of nucleotide sequence evolution can be evaluated in the jModelTest [15], which is a stand-alone extension of the popular program ModelTest. It provides a computational pipeline utilizing different existing programs for performing separate tasks. The evolutionary model best fitting with sequence data was determined using jModelTest version 2 which identified TIM2+I+G (transitional model $2+$ proportion of invariable site + rate heterogeneity) as the most appropriate model. Since MrBayes [16] does not implement the TIM2+I+G model, the second-best model GTR+I+G (general time reversible + proportion of invariable site + rate heterogeneity) was chosen for analysis. MrBayes is a widely used program for Bayesian inference of phylogeny and implements the Markov chain Monte Carlo (MCMC) method in various evolutionary models. The MCMC simulation was run in parallel for 1,000,000 generations and trees were sampled every 1,000 th generation in the MrBayes version $3.0 \mathrm{~b} 4$. A $50 \%$ majority rule consensus phylogenetic tree of the SRGAP2 gene was inferred from a post-burn-in posterior distribution of 751 trees. The inferred phylogenetic tree was tested for global and local molecular clocks using the likelihood ratio test (LRT) in HyPhy.

\section{Evolutionary Modeling of Neural Traits}

The growth of a biological trait is fundamentally multiplicative and, thus, species values were converted into a geometric domain using log-transformation. The measurements of 3 neural traits in 11 species of mammals were collected from the available literature (online suppl. table S2) [1]. The statistical modeling of evolution of a continuous trait along the branches of a phylogenetic tree is a powerful tool to understand the biological evolution [17]. 
Since closely related species share an evolutionary history, they, consequently, are not statistically independent as assumed in an ordinary least-squares regression. Thus, a phylogenetic general linear model was developed as an alternative method to introduce a correction of the phylogenetic signal in comparative data [17]. The trait evolution along the branches of the phylogenetic tree was modeled in the PGLS framework within the standard constant-variance random walk model in the subprogram CONTINUOUS which is included in the software package BayesTraits version 2.0 [17]. The BayesTraits is a software to analyze the evolution of a trait among a group of species with known phylogeny. Here, it is used to study the evolution of a continuous trait in a group of mammals. The program was used in an MCMC mode on the phylogenetic tree of the SRGAP2 gene. Rate deviation was set to maintain a parameter estimation acceptance rate between 20 and 40\%. This program calculates the logarithm of the harmonic mean of the likelihoods, which approximates marginal likelihood. The Bayes factor was calculated as follows: 2 (log [harmonic mean (better model)] - log [harmonic mean (worse model)]).

The correlation between 3 neural traits and the root-to-tip distance (i.e. substitution rate) estimated from the SRGAP2 gene phylogeny was examined under both the random walk (model A) and directional (model B) models of trait evolution. The root-to-tip distance represents a more inclusive evolutionary history of a species than individual terminal branches. The significance of results was determined based on the Bayes factor, which compares the marginal likelihood of two models. Model A was found to be the best model for all 3 neural traits. The dependence of a neural trait on the underlying phylogenetic tree was assessed by the Bayes factor, which compares a model estimating the $\lambda$ parameter freely with a model having lambda fixed at 1 . Similarly, $\kappa$ and $\delta$ were estimated for 3 neural traits using the same method. Three phylogenetic parameters, namely $\lambda$, $\kappa$ and $\delta$, were estimated for 3 neural traits. Parameters $\lambda$ and $\kappa$ measure the strength of the phylogenetic signal and gradual/punctuated evolution in the data, respectively. Parameter $\delta$ measures whether the rates of evolution have increased, decreased or remained constant over time.

The root-to-tip branch length was used as species data for regression analysis with the PGLS framework. The $\omega$ is a ratio between the number of nonsynonymous substitutions per nonsynonymous site $(\mathrm{dN})$ and the number of synonymous substitutions per synonymous site $(d S)$. The $\mathrm{dN} / \mathrm{dS}$ ratio $(\omega)$ was estimated using the CODEML as implemented in PAML (phylogenetic analysis by maximum likelihood) version 4 [18]. The PAML is the standard program based on maximum likelihood for testing evolutionary hypotheses like the role of selection in the evolution of a gene. The free-ratio model was applied to examine the variations in the $\mathrm{dN} /$ $\mathrm{dS}$ ratio across the mammalian lineages in the phylogenetic tree of the SRGAP2 gene. The rootto-tip $\omega, \mathrm{dN}$ and $\mathrm{dS}$ from the last common ancestor of mammals were estimated using the free-ratio branch model. The root-to-tip values of $\omega$, $\mathrm{dN}$ and $\mathrm{dS}$ were set as phenotypic values and were subjected to regression analysis in the PGLS framework. The significance of the regression coefficient was determined using the one-tailed t test. All statistical analyses were performed in the R language and environment [19].

\section{Ancestral State Reconstruction}

Ancestral state reconstruction was executed in the Mesquite version 3.04 software package for weighted squared-change parsimony [20] and the ape version 3.3 package [21] for the GLS framework in the R environment [19]. Ancestral states of the brain mass and the number of cortical neurons were reconstructed using the phylogeny inferred from the SRGAP2 gene. This approach permits the comparison of brain mass with the number of cortical neurons across 9 species of mammals, namely humans, chimpanzees, monkeys, dogs, elephants, horses, mice, rats and opossums. The age of ancestors in terms of million years ago (Mya) was estimated using Pathd8, which implements the mean path length rate-smoothing method without 
Table 1. Regression analyses of neural and phylogenetic traits in the PGLS framework

\begin{tabular}{lll}
\hline Analysis & $\mathrm{R}^{2}$ & $\mathrm{p}$ value \\
\hline Brain mass versus EQ & $\mathbf{0 . 4 5}$ & $\mathbf{0 . 0 1 3 2}$ \\
Brain mass versus number of cortical neurons & $\mathbf{0 . 8 7}$ & $\mathbf{2 . 6 2 \times 1 \mathbf { 1 } ^ { - 4 }}$ \\
EQ versus number of cortical neurons & 0.34 & 0.0528 \\
Brain mass versus branch length & 0.13 & 0.1384 \\
Number of cortical neurons versus branch length & $\mathbf{0 . 4 6}$ & $\mathbf{0 . 0 2 1 9}$ \\
Brain mass versus $\omega$ & 0.04 & 0.2777 \\
EQ versus $\omega$ & 0.03 & 0.3086 \\
Number of cortical neurons versus $\omega$ & 0.15 & 0.1497 \\
Number of cortical neurons versus dN & $\mathbf{0 . 3 8}$ & $\mathbf{0 . 0 3 7 4}$ \\
Number of cortical neurons versus dS & 0.27 & 0.0756 \\
\hline
\end{tabular}

The values and traits in bold indicate a significant association between traits.

any clock assumption [22]. The mean path length requires numerous reference nodes for time calibration, and at least one reference node is needed with an absolute age (fixed age) supplemented with either minimum age or maximum age. Molecular time estimates based on a large number of molecular clocks were used to specify the age of 3 fixed nodes (human-chimpanzee: 5.4 Mya; human-monkey: 23.3 Mya; human-gibbon: 14.9 Mya), 1 maximum node (human-cow: 98 Mya) and 1 minimum node (horse-cow: 82 Mya) [23].

\section{Results}

Statistical Dependence of Neural Traits on the SRGAP2 Gene Tree

Among 3 neural traits, both brain mass and number of cortical neurons showed a strong evidence that $\lambda=1$, indicating dependence of the brain mass and the number of cortical neurons on the underlying SRGAP2 gene tree (online suppl. tables S3-S6). Thus, the 64\% of variation in the brain mass $(\lambda=0.64)$ and the number of neurons $(\lambda=0.64)$ is consistent with random evolutionary changes along the branches of the SRGAP2 gene tree. However, only $55 \%$ of variation in EQ can be related to the phylogeny of the SRGAP2 gene. Both $\kappa$ and $\delta$ were equal to 1 , indicating gradual evolution of the brain mass and the number of cortical neurons in the mammalian lineage. However, EQ was estimated to have $\kappa<1(\kappa=0.35)$, indicating stasis in the longer branches, and to have $\delta=1(\delta=1.39)$, indicating gradual adaptations in mammals.

\section{Phylogenetically Controlled Regression Analyses}

Phylogenetically controlled regression analyses were performed among neural traits, such as log (brain mass), log (EQ) and log (number of cortical neurons) along with traits derived from phylogeny of the SRGAP2 gene, such as log (root-to-tip branch length), log (rootto-tip $\omega$ ), $\log$ (root-to-tip dN) and $\log$ (root-to-tip dS) in BayesTraits. Only 4 out of 10 phylogenetically controlled regression analyses showed a significant association (table 1). A significant positive association was found between brain mass and number of cortical neurons $\left(R^{2}=0.87 ; n=9 ; p=2.62 \times 10^{-4} ;\right.$ fig. $\left.1 \mathrm{a}\right)$ and brain mass and $E Q\left(R^{2}=0.45 ; n=11 ; p=0.0132\right)$, whereas EQ and number of cortical neurons had a nonsignificant association $\left(R^{2}=0.34 ; n=\right.$ $9 ; p=0.0528$ ). The data for mammals are evenly distributed along the regression line, with 4 species, namely humans, chimpanzees, rats and mice, being above the regression line, and 4 species, namely opossums, dogs, horses and elephants, being below the regression line 
Fig. 1. a Bivariate logarithmic plot between the brain mass and cortical neurons. b Rate of substitutions in the SRGAP2 gene and number of cortical neurons. c Rate of nonsynonymous substitutions in the SRGAP2 gene and number of cortical neurons. Bestfit lines are least-squares regression lines fitted to the raw data (green line) and the phylogenetically controlled regression line (red line).
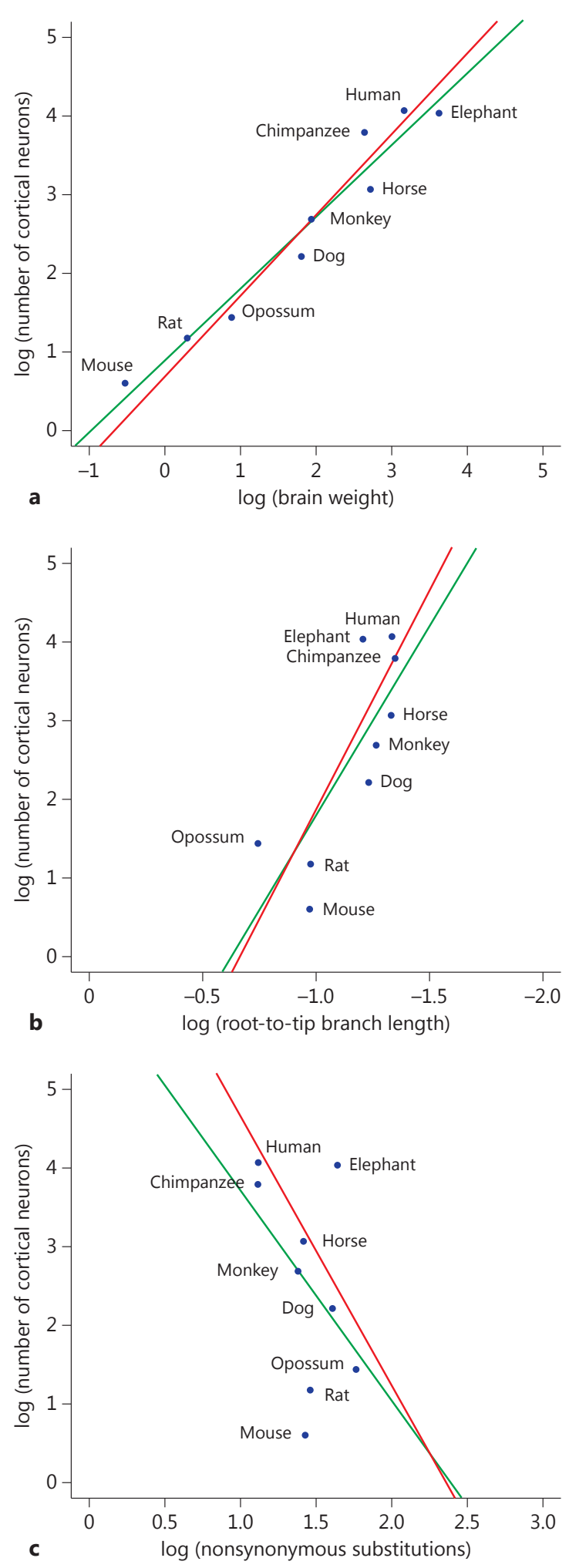


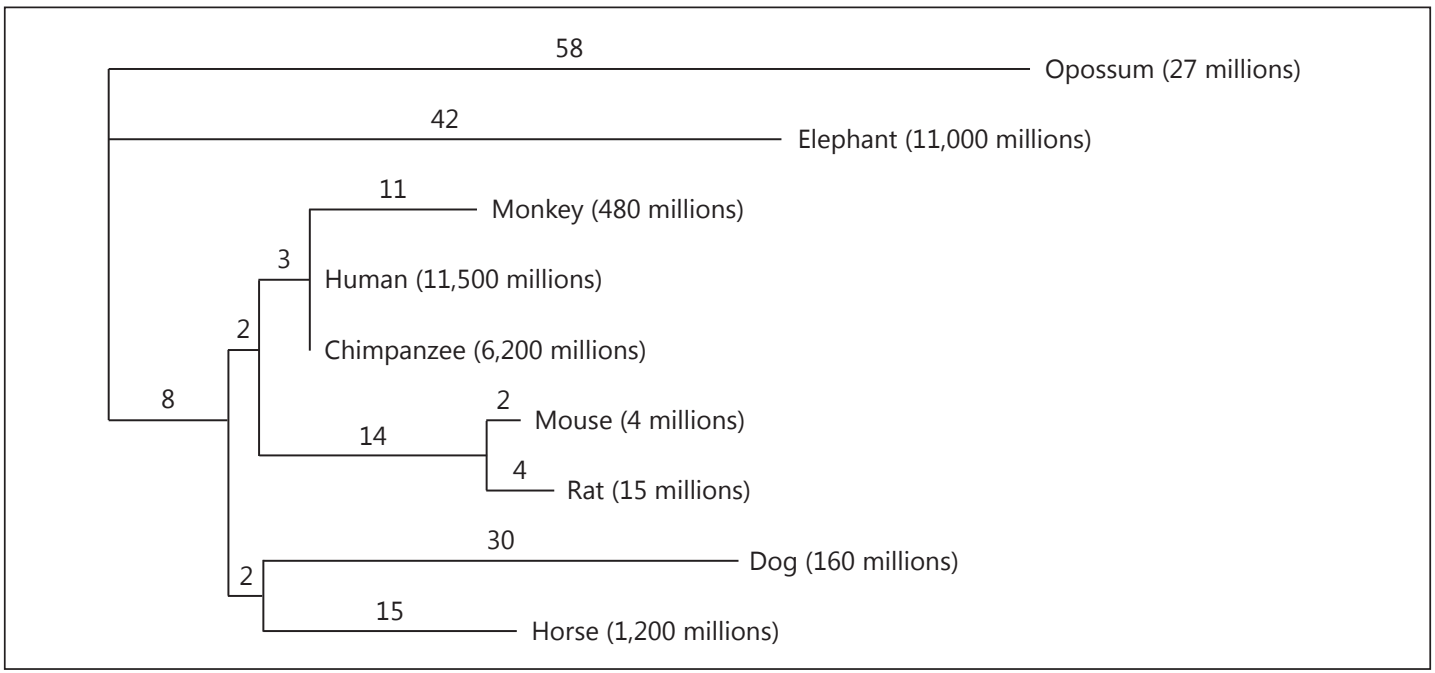

Fig. 2. The phylogeny of the SRGAP2 gene based on nonsynonymous substitutions showing the number of neurons in each species (in parentheses) and the total number of nonsynonymous substitutions along each branch (number above each branch).

(fig. 1a). The neuron number of monkeys falls on the regression line, suggesting that monkeys have a neuron number expected for a mammal of that brain mass. The number of cortical neurons are positively correlated with the branch length $\left(R^{2}=0.46 ; n=9 ; p=0.0219\right.$; fig. $\left.1 b\right)$, indicating an increase in the rate of nucleotide substitution in the SRGAP2 gene along with the accelerated evolution of neuron numbers in the cortex of mammals.

\section{Selective Constraints on the SRGAP2 Gene}

The number of cortical neurons was negatively associated $\left(R^{2}=0.38 ; n=9 ; p=0.0374\right.$; fig. 1c) with the root-to-tip nonsynonymous substitution per nonsynonymous site ( $\mathrm{dN})$ in each species, suggesting a decline in the rate of nonsynonymous substitutions with a corresponding increase in the neuron number in mammals. The $\mathrm{dN} / \mathrm{dS}$ ratio $(\omega)$ for SRGAP2 is found to be significantly $<1$ (mean $=0.1416$; $S D=0.0457$ ) in all mammalian lineages, indicating that the SRGAP2 gene is under the evolutionary pressure of purifying selection. However, the average $\omega(0.8513)$ across hominoids is not significantly different from the average value of other mammalian lineages $\left(\omega=0.0565 ; \chi^{2}\right.$ test; LRT $\left.=3.7 ; p=0.1737\right)$. Similarly, the average $\omega$ for the human-chimpanzee lineage $(0.001)$ is not significantly different either from the average $\omega$ value in other lineages $\left(\omega=0.0571 ; \chi^{2}\right.$ test; LRT $\left.=3.22 ; p=0.0727\right)$. Interestingly, elephant, opossum and rodent lineages are subjected to significantly different selection pressures in comparison to other lineages. The $\omega$ for the elephant lineage $(\omega=$ $0.1531)$ is significantly higher than the average $\omega$ of other mammals $\left(\omega=0.0500 ; \chi^{2}\right.$ test; LRT $=19.98 ; p=7.82 \times 10^{-6}$ ), indicating a lower intensity of the purifying selection in elephants. In contrast, the opossum lineage $(\omega=0.0393)$ has significantly lower $\omega$ values than the average of other lineages $\left(\omega=0.0642 ; \chi^{2}\right.$-test; LRT $\left.=7.82 ; p=5.16 \times 10^{-3}\right)$. Similarly, the rodent lineage $(\omega=0.0248)$ has a lower average $\omega$ value than the average $\omega$ of the rest of the mammals $\left(\omega=0.0616 ; \chi^{2}\right.$ test; LRT $\left.=11.16 ; \mathrm{p}=8.35 \times 10^{-4}\right)$. The total number of nonsynonymous substitutions on the SRGAP2 gene in relation to the neuron number showed an interesting pattern across mammalian lineages (fig. 2). Humans and chimpanzees maintained the status quo as far as nonsynonymous substitutions on the SRGAP2 gene are concerned but had undergone a substantial increase in the neuron number in their brain cortex. Monkeys accu- 
Table 2. Ancestral state reconstruction of the brain mass and cortical neuron numbers in mammals using GLS

\begin{tabular}{lccc}
\hline Last common ancestor & Estimate of brain mass, g & Estimate of neuron number & $\begin{array}{c}\text { Age, } \\
\text { Mya }\end{array}$ \\
& & & 5.4 \\
\hline Human-chimpanzee & $679.69(679.64-679.74)$ & $7,485.71(7,485.65-7,485.76)$ & 23.3 \\
Hominoids-monkey & $391.84(391.73-391.95)$ & $5,212.02(5,211.90-5,212.14)$ & 32.29 \\
Rat-mouse & $65.88(65.70-66.06)$ & $443.54(443.37-443.72)$ & 79.29 \\
Rodents-primates & $525.65(525.47-525.83)$ & $3,526.56(3,526.38-3,526.74)$ & 91.58 \\
Horse-dog & $579.44(579.27-579.61)$ & $2,326.58(2,326.40-2,326.75)$ & 98 \\
Euarchontoglires-Ferungulata & $681.47(681.32-681.63)$ & $2,919.83(2,919.68-2,919.98)$ & 98 \\
\hline
\end{tabular}

Figures in parentheses indicate 95\% confidence intervals of the estimate. Age estimates in million years ago (Mya) are obtained by the mean path length method.

mulated 11 nonsynonymous substitutions on the SRGAP2 gene after their divergence from the last common ancestor of primates. The total number of nonsynonymous substitutions accumulated on the SRGAP2 gene in the primate lineage (mean $=16.66$; $\mathrm{SD}=6.35$ ) is significantly smaller than that in other mammalian lineages under study (mean $=36.5 ; \mathrm{SD}=12.80$; two-sample Kolmogorov-Smirnov test; $\mathrm{D}=1 ; \mathrm{p}=0.03663$ ). This indicates that the SRGAP2 gene was under selective constraint in the primate lineage, especially in the human-chimpanzee branch.

\section{Increase and Decrease of Neural Traits in Ancestors}

Ancestral state reconstruction of brain mass and neuron number using the GLS framework was applied to document changes in 2 neural traits, namely brain mass and the number of cortical neurons, through mammalian evolution along with the estimated age of each ancestor (table 2). The last common ancestor of Euarchontoglires (ancestors of primates and rodents) and Ferungulata (ancestors of artiodactyls and carnivores) had a brain mass of $681.47 \mathrm{~g}$. The brain mass initially decreased in the rodent/primate common ancestor (525.65 g) and the horse/dog common ancestor (579.44 g). Subsequently, it decreased further in the old world monkey-ape common ancestor (391.84 g) and decreased several fold in the rodent common ancestor (65.88 g). However, the brain mass increased twofold in the human/chimpanzee common ancestor (679.69 g) and subsequently more than twofold in the human lineage $(1,450 \mathrm{~g})$. The chimpanzee lineage had undergone a $63 \%$ decrease in the brain size (430 g) from the last human/chimpanzee common ancestor.

The neuron number in the cortex of the last common ancestor of Euarchontoglires and Ferungulata was estimated to be 2,920 millions. There is a continual increase in the neuron number through the primate lineage but a decline in neuron number across the rodent lineage and the dog-horse lineage. Both neural traits showed interesting trends when the ancestral state was reconstructed through maximum parsimony and compared using mirror phylogeny (fig. 3). Opossums maintained a low brain mass and a low number of neurons like the common ancestor of metatherian and eutherian mammals. The elephant lineage showed a maximum increase in brain mass followed by the human lineage. Conversely, the human lineage showed a maximum increase in the neuron number in the cortex followed by the elephant lineage.

\section{Local Clocks in Ape and Rodent Lineages}

The inferred phylogenetic tree was tested for the global molecular clock, and the null hypothesis of the global molecular clock was rejected using the LRT in HyPhy. However, the 


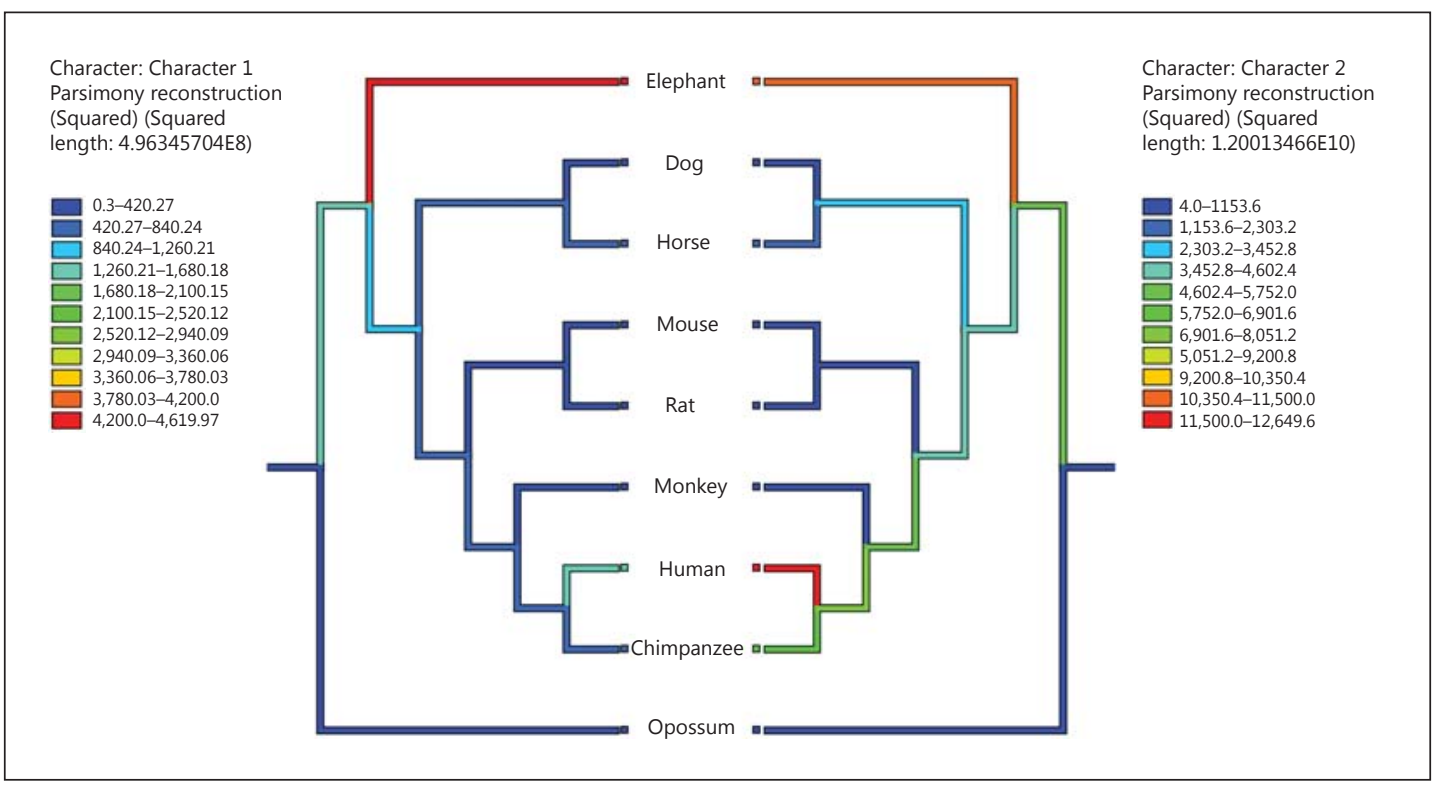

Fig. 3. Mirror phylogeny of the SRGAP2 gene in mammals showing maximum-parsimony ancestral state reconstruction of the brain mass (left side) and the neuron number in the cortex (right side). Major increases have occurred independently in human and elephant lineages. The side bar shows the color legend for neural trait values mapped onto the SRGAP2 gene tree.

null hypothesis of the local molecular clock could not be rejected for 3 lineages, namely human-chimpanzee (LR statistic $=2.72 ; \mathrm{p}=0.255$ ), human-chimpanzee-gibbon (LR statistic $=2.98 ; p=0.560)$ and mouse-rat (LR statistic $=0.85 ; p=0.654)$. The rest of the lineages did not follow the local molecular clock in their evolutionary history, and the null hypothesis of local clock was rejected for all these lineages at a high significance level $(p<0.01)$.

\section{Discussion}

The result indicates that the SRGAP2 gene may have a significant role in the progression of overall brain size in mammals. These findings also provide strong evidence that the evolution of the SRGAP2 gene is intimately linked to the expansion of neuron numbers in the brain cortex of mammals. The brain mass and neuron number in the cortex are linearly correlated in mammals, and this finding is consistent with the earlier findings in primate brains [8]. The ancestral $S R G A P 2$ gene in all mammalian lineages is subjected to a strong purifying selection in order to maintain the functional role of the gene. The deleterious mutations within a functional gene are amenable to a purifying selection and less likely to be fixed in a population. Thus, the signature of the purifying selection is imprinted on a gene in form of a substantial reduction in the amino acid substitution process. Moreover, the increase in the neuron number is negatively correlated with the substitution rates and nonsynonymous changes in different mammalian lineages. The stasis in terms of nonsynonymous changes indicates that the structure of the SRGAP2 protein was constrained in the last common ancestor of humans and chimpanzees. It seems that the natural selection has opted for an optimal protein structure of SRGAP2 that can carry out a specific function to maintain not only the maximal number of neurons but also the maximum size of the brain. However, the extraordinary increase in the brain size and neuron number in humans has been attributed 
to the novel copy (SRGAP2C) of the SRGAP2 gene [9]. It appears that SRGAP2A continues to maintain its ancestral function in humans, and the new duplicate, namely $S R G A P 2 C$, adopts a new function in humans and mediates the accelerated growth of the brain and the number of cortical neurons. This study clearly indicates that the ancestral SRGAP2 gene did play a very crucial role in the evolution of the brain size and the neuron number throughout the mammalian evolution. Therefore, the key role of the ancestral SRGAP2 gene in the manifestation of a large brain with a large number of neurons in the primate lineage cannot be ruled out before its duplication in the human lineage. In the human lineage, the novel SRGAP2C gene accumulated 7 amino acid substitutions within a short span of 4 million years after duplication from a highly constrained ancestral gene, SRGAP2A [10]. The relaxation of selective pressure on the SRGAP2C gene might have played a crucial role in the manifestation of some of the human-specific high-level cognitive functions in the human lineage. The SRGAP2C protein is known to heteromerize with its ancestral protein SRGAP2A resulting in an antagonistic function equivalent to the knock-out of the ancestral gene [11]. It will be interesting to know whether any other brain-specific gene is interacting with this novel copy of SRGAP2C in a synergistic or antagonistic manner. It can be speculated that the human brain would not have reached this extraordinary level in terms of intelligence in the absence of this novel duplicated gene. Therefore, further evolutionary studies on the other novel duplicate genes in humans along with their ancestral paralogous genes in mammals will unveil the genomic basis of extraordinary human intelligence. The signature of local molecular clocks in ape and rodent lineages indicates that the constant rate of substitution in the SRGAP2 gene in two distantly related lineages is shaped by various selective forces.

The major increase in the brain size and neuron number in two distant lineages, namely humans and elephants, is an example of parallel and convergent patterns of adaptive phenotypic evolution. This hypothesis can be supported by the fact that aerobic energy metabolism genes in the brain evolved adaptively in human and elephant lineages in addition to the presence of other common traits, such as large brain, high intelligence, social bond, long gestation period and long life span [24]. The elephant has a large brain size with a maximum number of neurons (257 billions); nevertheless, the cerebral cortex of elephants contains only about one third of the number of cortical neurons present in humans [25]. Although elephants have a lower number of cortical neurons, they exhibited many advanced cognitive abilities which they share with humans, such as insightful problem solving and tool use [26]. The evolution of shared neural traits in humans and elephants might be due to regular interactions and frequent conflicts between two distantly related species in a common ecological niche throughout their evolutionary history. The drastic difference in the brain size and the number of cortical neurons between humans and chimpanzees, despite maintaining an identical sequence structure by the SRGAP2 gene and encoded protein, may be attributed to the altered gene expression or expression of the duplicated SRGAP2C gene in humans.

The presence of a large brain in the human-chimpanzee common ancestor and a reduction in the brain size and neuron numbers in the derived early hominids and the recent chimpanzee lineages is not consistent with available fossil evidences. It clearly indicates that the brain size and neuron numbers were considerably reduced in the ancestral human lineage (320-380 $\mathrm{cm}^{3}$ in Sahelanthropus) [27] as well as in the chimpanzee lineage. The fossil evidences suggested that there was a substantial decrease in endocranial volume in recent human populations in different parts of the world during the last 10,000 years [28]. It has been argued that the reduction in body size may be the primary cause for this decrease in the brain size [29]. Therefore, the human-chimpanzee common ancestor might have a larger body size with a comparable brain size and number of cortical neurons. However, this study indicates that a considerable decrease in the brain size and body size in both human and chimpanzee lineages occurred after the split, probably due to existing environmental condi- 
tions and other genetic factors. The molecular findings of the brain size are not consistent with fossil records due to the fact that the brain size reported from fossil records may not be accurate estimates of the brain size in the common ancestor of human and chimpanzee. The estimates from crushed or incomplete fragments of fossil records are prone to inaccuracy in measurement with a large margin of error.

The rate of amino acid substitution during protein evolution is guided by the stringency of functional constraint. Proteins with a rigorous function are likely to accumulate a less number of amino acid changes during their evolutionary history and consequently evolve slower than proteins with a weaker functional constraint. There are many examples of evolutionary constraints on genes linked with the stringency of their function. Essential genes of bacteria evolve slower than nonessential genes [30] and house-keeping genes are known to be under stronger selective constraint than tissue-specific genes [31]. Similarly, the genes coding for proteins involved in a common physiological function are likely to evolve in a correlated manner [32]. Therefore, the correlation between the evolution of a phenotypic trait and the evolution of a gene suggests a key role of the SRGAP2 gene in the manifestation of a phenotypic trait (i.e. number of neurons in the cortex) in mammals. Further studies on this gene and the interaction partners with a more elaborate neural trait dataset will give us new insights into the mechanistic role of the gene in the rapid increase in intelligence in mammals.

\section{Acknowledgements}

The author thanks the Department of Biotechnology (DBT), Govt. of India, New Delhi for financial support to the Centre for Bioinformatics, Pondicherry University.

\section{Disclosure Statement}

The author declares no conflicts of interest.

\section{References} cognition in healthy elderly men. Neurology 2002;59:169-174.

4 Jung RE, Haier RJ: The parieto-frontal integration theory (P-FIT) of intelligence: converging neuroimaging evidence. Behav Brain Sci 2007;30:135-154.

5 Deaner RO, Isler K, Burkart J, van Schaik C: Overall brain size, and not encephalization quotient, best predicts cognitive ability across non-human primates. Brain Behav Evol 2007;70:115-124.

6 Russell DA: Exponential evolution: implications for intelligent extraterrestrial life. Adv Space Res 1983;3: 95-103.

7 Pakkenberg B, Gundersen HJG: Neocortical neuron number in humans: effects of sex and age. J Comp Neurol 1997;384:312-320.

8 Herculano-Houzel S, Collins CE, Wong P, Kaas JH: Cellular scaling rules for primate brains. Proc Natl Acad Sci USA 2007;104:3562-3567.

9 Cairo O: External measures of cognition. Front Hum Neurosci 2011;5:108.

10 Dennis MY, Nuttle X, Sudmant PH, Antonacci F, Graves TA, Nefedov M, Rosenfeld JA, Sajjadian S, Malig M, Kotkiewicz H, Curry CJ, Shafer S, Shaffer LG, de Jong PJ, Wilson RK, Eichler EE: Evolution of human-specific neural SRGAP2 genes by incomplete segmental duplication. Cell 2012;149:912-922.

11 Guerrier S, Coutinho-Budd J, Sassa T, Gresset A, Jordan NV, Chen K, Jin W-L, Frost A, Polleux F: The F-BAR domain of srGAP2 induces membrane protrusions required for neuronal migration and morphogenesis. Cell 2009;138:990-1004. 
Tiwary: Evolution of the SRGAP2 Gene Is Linked to Intelligence in Mammals

12 Guo S, Bao S: srGAP2 arginine methylation regulates cell migration and cell spreading through promoting dimerization. J Biol Chem 2010;285:35133-35141.

13 Montgomery SH, Mundy NI: Evolution of ASPM is associated with both increases and decreases in brain size in primates. Evolution 2011;66:927-932.

14 Pond SLK, Muse SV, Frost SDW: HyPhy: Hypothesis Testing Using Phylogenies. San Diego, University of California, 2004.

15 Posada D: jModelTest: phylogenetic model averaging. Mol Biol Evol 2008;25:1253-1256.

16 Ronquist F, Teslenko M, van der Mark P, Ayres DL, Darling A, Höhna S, Larget B, Liu L, Suchard MA, Huelsenbeck JP: MrBayes 3.2: efficient Bayesian phylogenetic inference and model choice across a large model space. Syst Biol 2012;61:539-542.

17 Pagel M: Inferring the historical patterns of biological evolution. Nature 1999;401:877-884.

18 Yang Z: PAML 4: phylogenetic analysis by maximum likelihood. Mol Biol Evol 2007;24:1586-1591.

19 R Development Core Team: R: A Language and Environment for Statistical Computing. Vienna, R Foundation for Statistical Computing, 2015.

20 Maddison WP, Maddison DR: Mesquite: a modular system for evolutionary analysis. Version 3.04. 2015. http://mesquiteproject.org.

21 Paradis E, Claude J, Strimmer K: APE: analyses of phylogenetics and evolution in R language. Bioinformatics 2004;20:289-290.

22 Britton T, Anderson CL, Jacquet D, Lundqvist S, Bremer K: Estimating divergence times in large phylogenetic trees. Syst Biol 2007;56:741-752.

23 Hedges SB, Kumar S: Genomic clocks and evolutionary timescales. Trends Genet 2003;19:200-206.

24 Herculano-Houzel S, Avelino-de-Souza K, Neves K, Porfírio J, Messeder D, Calazans I, Mattos L, Maldonado J, Manger PM: The elephant brain in numbers. Front Neuroanat 2014;8:46.

25 Goodman M, Sterner KN, Islam M, et al: Phylogenomic analyses reveal convergent patterns of adaptive evolution in elephant and human ancestries. Proc Natl Acad Sci USA 2009;106:20824-20829.

26 Foerder P, Galloway M, Barthel T, Moore DE III, Reiss D: Insightful problem solving in an Asian elephant. PLoS One 2011;6:e23251.

27 Brunet M, Guy F, Pilbeam D, et al: A new hominid from the Upper Miocene of Chad, Central Africa. Nature 2002; 418:145-151.

28 Bednarik RG: Doing with less: hominin brain atrophy. Homo 2014;65:43-449.

29 Ruff CB, Trinkaus E, Holliday TW: Body mass and encephalization in Pleistocene Homo. Nature 1997;387: 173-176.

30 Jordan IK, Rogozin IB, Wolf YI, Koonin EV: Essential genes are more evolutionary conserved than are nonessential genes in bacteria. Genome Res 2002;12:962-968.

31 Zhang L, Li W-H: Mammalian housekeeping genes evolve more slowly than tissue-specific genes. Mol Biol Evol 2004;21:236-239.

32 Tiwary BK: Correlated evolution of gonadotropin-releasing hormone and gonadotropin-inhibitory hormone and their receptors in mammals. Neuroendocrinology 2013;97:242-251. 